\title{
Setup and optimization of primer for protease gene of human immunodeficiency virus-I as evaluation drug resistance manner in Iranian patients
}

\begin{abstract}
Human immunodeficiency virus (HIV-1) is a problem of global health. After the development of anti-retroviral therapy, the mortality and morbidity rate was reduced in whom was infected with HIV-1. However, because of many reasons such as virologic, immunologic, HAART regimen has a different result in HIV-1 infected patients. CD4 T Lymphocyte count and viral load test confirmed and showed that in some patients have treatment failure and need to analysis HIV-1 drugs target sequence and review in the administration of HAART regimen. There is some commercial kit for HIV-1 genotyping. Beside of these commercial kits, ANRS designed and released primer sets for amplification and sequencing of the HIV-1 genes such as Pol, Env. In the present study, protease sequence in the blood samples of 20 patients was amplified and investigated with in-house developed primer. These protease sequences were not amplified by ANRS primers.
\end{abstract}

Keywords: hiv-1, primer, PCR, ANRS, drug resistance
Volume 2 Issue 3 - 2017

\author{
Mohammad Gholami,' Negin Hosseini \\ Rouzbahani,,'2 KatayounTayeri,' Reza \\ AdITabatabai,' Minoo Mohraz' \\ 'Iranian Research Center for HIV AIDS, Tehran University of \\ Medical Science, Iran \\ ${ }^{2}$ Department of Immunology, Tehran University of Medical \\ Sciences, Iran
}

Correspondence: Minoo Mohraz, Iranian Research Center for HIV AIDS (IRCHA) Iranian Institute for Reduction of High-Risk Behaviors, Tehran University of Medical Science,

EmailM.Mohraz@iran-ccm.ir

Received: April 27, 2017| Published: May 08, 2017

\section{Introduction}

In the early 1980s, a new disease emerged among Men who have sex with men (MSM). This unknown origin infection was followed by the unusual opportunistic illnesses along with rare malignancies such as Kaposi sarcoma, lymphoma. ${ }^{1,2}$ Human immunodeficiency virus type $1(\mathrm{HIV}-1)$ was known as a predisposing factor for opportunistic infection in homosexual patients., 3 By medical management of HIV-1 infected patients with antiretroviral drugs, replication of HIV-1 significantly can receive to the undetectable levels. ${ }^{5,6}$ There is a different kind of approved antiretroviral medicines available to prescribe in the world. ${ }^{7}$ About $10^{4}$ to $10^{5}$ copy $/ \mathrm{ml}$ virus present in plasma samples derived from naïve/untreated patients. ${ }^{8}$ Reverse transcriptase (RT) of HIV-1, RNA virus, has no proofreading ability. This error-prone transcription activity can generate one to 10 mutations in each viral replication cycle..$^{9}$ Antiretroviral therapy considerably decreases selection of virus clones. ${ }^{10}$ Increasing the viral load up to $1000 \mathrm{copy} / \mathrm{ml}$ is a sign of treatment failure and evaluation of drug resistance pattern is essential in the patients who are under HAART treatment.

There are many commercial HIV genotyping kits for evaluation of drug resistancy. ${ }^{11-13}$ However, these kind of commercial kits are expensive, less sensitive to non-B subtypes and they are not accessible in some countries. For these reasons, some reports have suggested setup and optimization in-house kit for evaluation mutation that occurs in the genome of virus. ${ }^{14-16}$

\section{Material and methods}

\section{Primer design}

The HIV-1 Reference sequences were downloaded from HIV database. A set of primer were designed and validated within the region of the protease gene of HIV-1 (from codon 1-95) by oligo7 and primer-primer 6 softwares Table 1. At first, the sequence of primer bioinformatically analyzed in NCBI database in order to evaluation of specifying of primers. Finally, the designed primers were synthesized by Macrogen Company (South Korea).

Table I Sequence of forward and revers primer

Forward primer sequence CTCAAATCACTCTTTGGCAACG

Reverse primer sequence CATTCCTGGCTTTAAYBTTACTGG

\section{Sample collection}

A blood sample was taken from HIV each patients who were referred to Iranian research center for HIV and AIDS (IRCHA) for drug resistance test. A plasma sample from each patient was collected into the defined tube and stored in -70c until extraction HIV RNA.

\section{RNA extraction}

RNA extraction performed with QIAamp ${ }^{\circledR}$ Viral RNA kit (Qiagen, Germany according to the protocol of kit). After RNA extracted, RNA kept in -70c till PCR reaction.

\section{Polymerase chain reaction}

Target sequence amplified with Qiagen one-step RT-PCR kit according to the following gradient cycle, $50^{\circ} \mathrm{C}$ 50min (cDNA synthesis), and $95^{\circ} \mathrm{C} 15 \mathrm{~min}$. PCR reaction performed with $95^{\circ} \mathrm{C}$ $30 \mathrm{sec}, 60^{\circ} \mathrm{C} 45 \mathrm{sec}$, and $72^{\circ} \mathrm{C} 1 \mathrm{~min}$ for 40 cycles with Bio-Rad PCR machine Table 1 . The PCR product analyzed with $1 \%$ gel agarose along with $100 \mathrm{bp}$ ladder (thermo life technology) and then sequenced via Sanger Sequencing method (Macrogen Company, South Korea). The results of sequencing analyzed in Stanford HIV drug resistant database for evaluation of drug resistance pattern.

\section{Results}

Analysis of primer sequence in 23 reference sequence confirmed that this primer know all this sequence especially CRF-AD35 which 
is dominant subtype among Iranian patients (Figure $1 \&$ Figure 2). $18 / 20$ patients who have viral load above 1000 copy/ml that they couldn't amplify with ANRS protease primer was amplified with this set primer and PCR products analyzed on $1 \%$ gel agarose and sequenced by Sanger sequencing method (Figure $3 \&$ Figure 4 ).

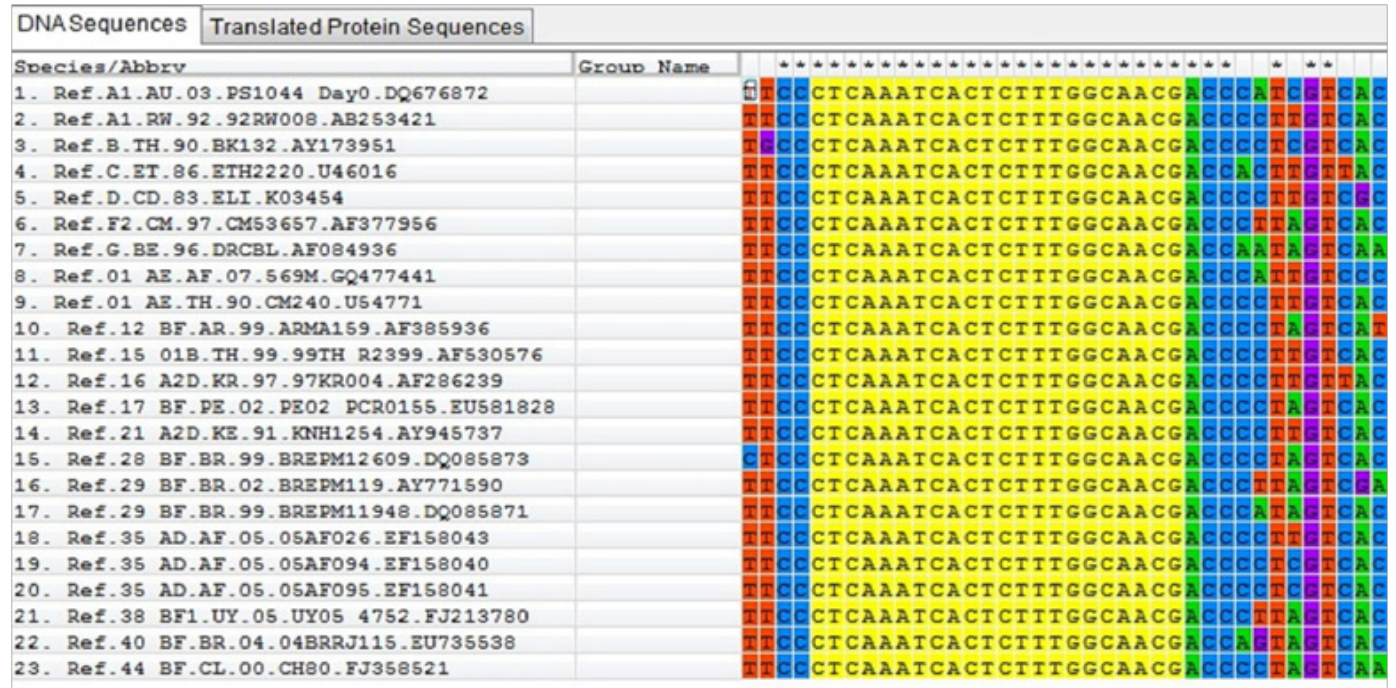

Figure I HIV-I forward primer location.

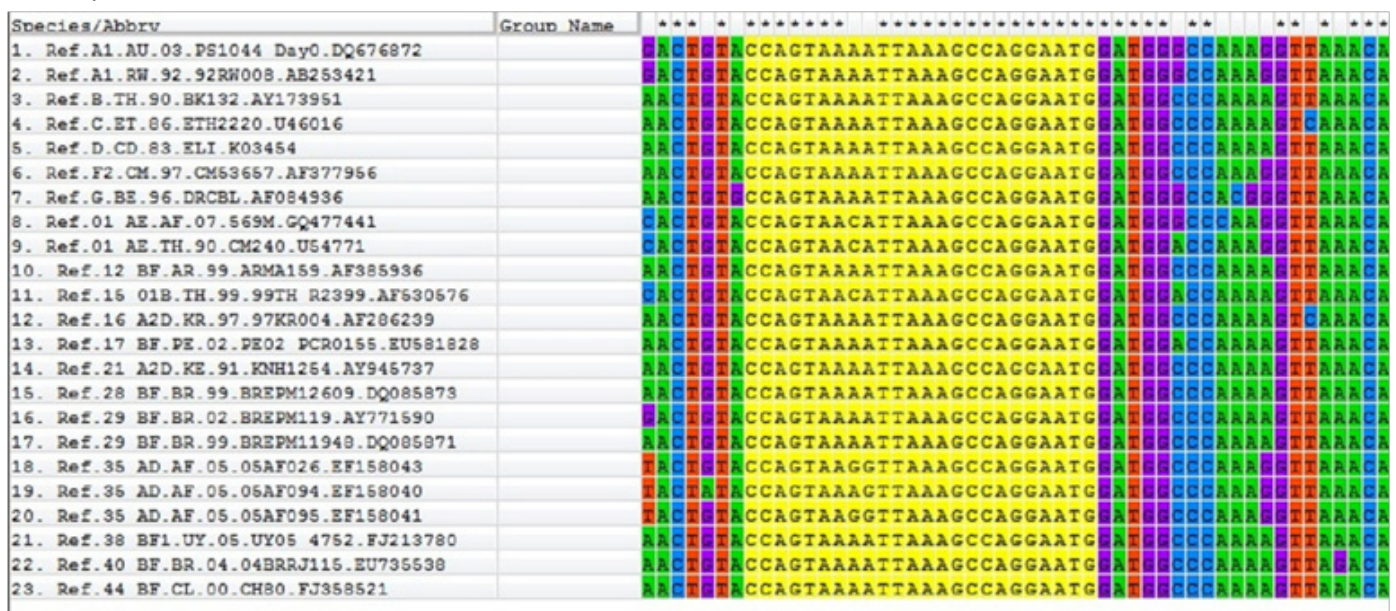

Figure 2 HIV-I revers primer location.

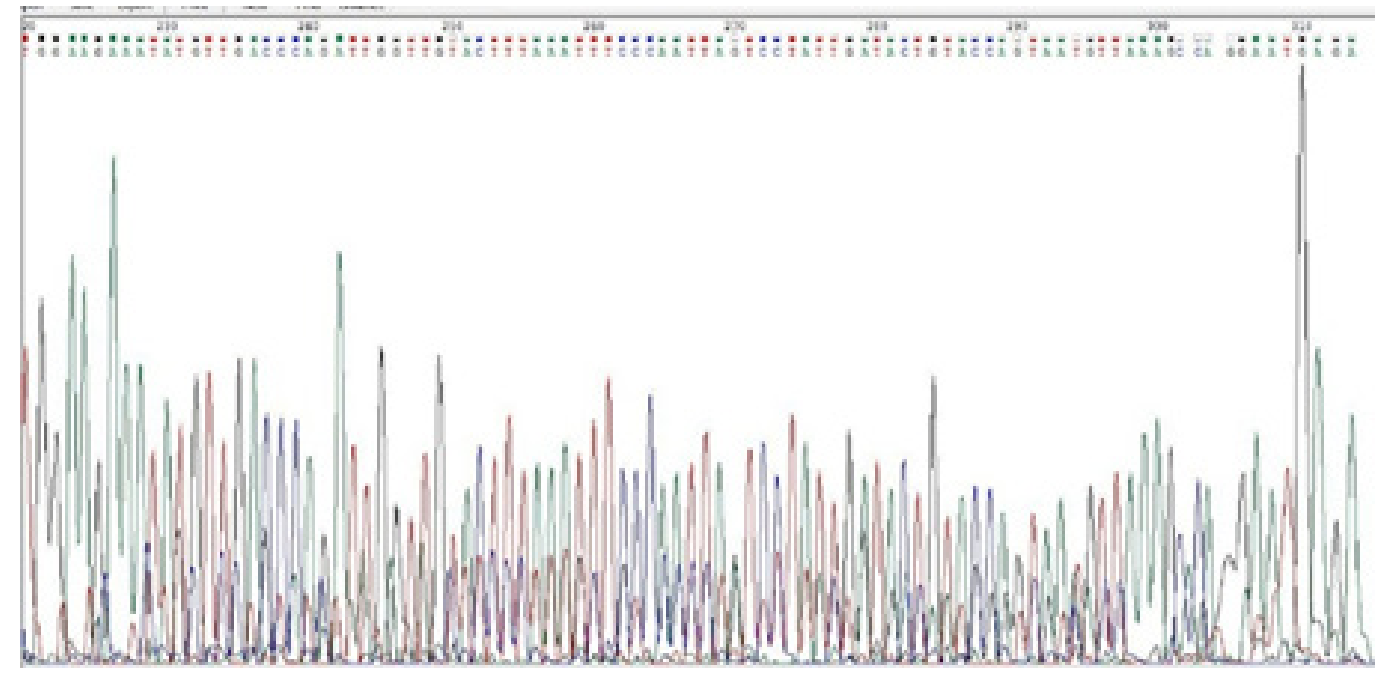

Figure 3 Sequencing result.

Citation: Gholami M, Rouzbahani NH, Tayeri K, et al. Setup and optimization of primer for protease gene of human immunodeficiency virus-I as evaluation drug resistance manner in Iranian patients. Int J Mol Biol Open Access. 2017;2(3): 10 I-103. DOI: 10.15406/ijmboa.2017.02.00023 


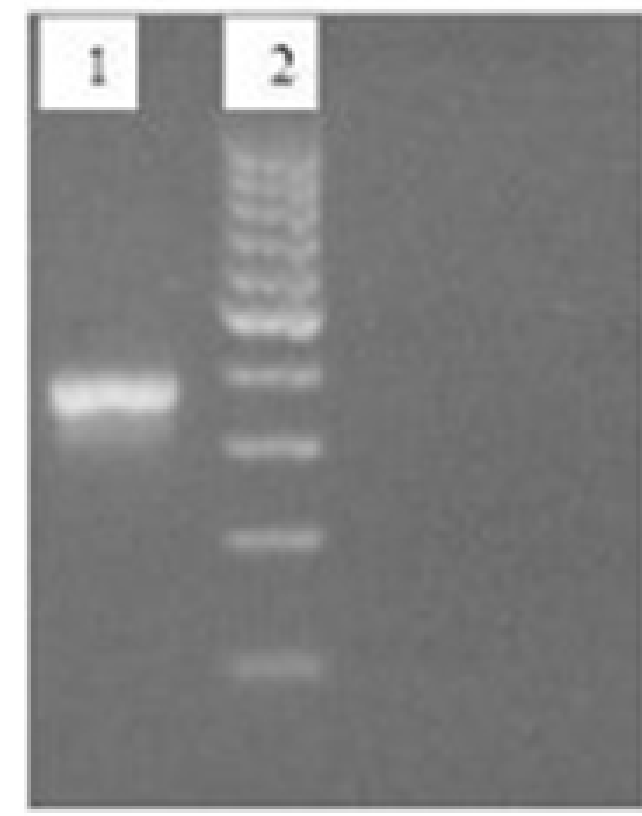

Figure $4 \mathrm{Gel}$ agarose result, lanel, 344bp specific band and lane 2, 100pb ladder.

\section{Discussion}

When the first HIV-1pandemic occurred (group M) nearly 60million people were infected and more than twenty-five million of infected patients were died. ${ }^{16}$ The combination of retroviral therapy, HAART, had significantly reduced morbidity and mortality rate that was associated with HIV-1 infection ${ }^{17,18}$ However, for many reasons such as virologic, immunologic and clinical responses patients are at risk for treatment failure. ${ }^{19}$ Virology failure may be occur because of lack of response to HAART or mutation that occur in the present of drug pressure. Evaluation of drug resistancy is important for management and fallow-up of infected patients. ${ }^{20}$ There is some in-house developed method for amplification and analysis of HIV sequence in the patients who have treatment failure. ${ }^{15}$ In our study, 20 patients have treatment failure and did not amplify by ANRS protease primer. ${ }^{15}$ Based on our results, 18 of 20 were amplified with our in-house designed set primer by one round PCR reaction instead of nested PCR. Finally, Sensitivity of this primers is more than ANRS protease primer. It may be because of special Iranian dominant subtype i.e. CRF-AD $35^{21}$ and our primer specially designed for this kind of subtype.

\section{Acknowledgements}

None.

\section{Conflict of interest}

Author declares that there is no conflict of interest.

\section{References}

1. Hart C, Beeching N, Duerden B. Infections in AIDS. Journal of medical microbiology. 2000;49(11):947-967.

2. Greene WC. A history of AIDS: looking back to see ahead. Eur J Immunol. 2007;37(S1):S94-S102.
3. Gallo RC, Salahuddin SZ, Popovic M, et al. Frequent detection and isolation of cytopathic retroviruses (HTLV-III) from patients with AIDS and at risk for AIDS. Science. 1984;224(4648):500-503.

4. Sharp PM, Hahn BH. Origins of HIV and the AIDS pandemic. Cold Spring Harb perspect in med. 2011;1(1):a006841.

5. Autran B, Carcelain G, Li TS, et al. Positive effects of combined antiretroviral therapy on CD4+ T cell homeostasis and function in advanced HIV disease. Science. 1997;277(5322):112-116.

6. Arts EJ, Hazuda DJ. HIV-1 antiretroviral drug therapy. Cold Spring Harb perspect in med. 2012;2(4):a007161.

7. Hirsch MS, Brun-Vézinet F, Richard T, et al. Antiretroviral drug resistance testing in adult HIV-1 infection: recommendations of an International AIDS Society-USA Panel. JAMA. 2000;283(18):2417-2426.

8. Ledergerber B, Egger M, Opravil M, et al. Clinical progression and virological failure on highly active antiretroviral therapy in HIV-1 patients: a prospective cohort study. Lancet. 1999;353(9156):863-868.

9. Abram ME, Ferris AL, Shao W, et al. Nature, position, and frequency of mutations made in a single cycle of HIV-1 replication. J Virol. 2010;84(19):9864-9878.

10. Stengel RF. Mutation and control of the human immunodeficiency virus Math Biosci. 2008;213(2):93-102.

11. Eshleman SH, Hackett J, Swanson P, et al. Performance of the Celera Diagnostics ViroSeq HIV-1 Genotyping System for sequence-based analysis of diverse human immunodeficiency virus type 1 strains. J Clin Microbiol. 2004;42(6):2711-2717.

12. Maes B, Schrooten Y, Snoeck J, et al. Performance of ViroSeq HIV-1 Genotyping System in routine practice at a Belgian clinical laboratory. Journal of virological methods. 2004;119(1):45-49.

13. Bennett DE, Bertagnolio S, Sutherland D, et al. The World Health Organization's global strategy for prevention and assessment of HIV drug resistance. Antiviral therapy. 2008;13(2):113.

14. Bennett DE, Camacho RJ, Otelea D, et al. Drug resistance mutations for surveillance of transmitted HIV-1 drug-resistance: 2009 update. PloS one. 2009;4(3):e4724.

15. Zhou Z, Wagar N, DeVos JR, et al. Optimization of a low cost and broadly sensitive genotyping assay for HIV-1 drug resistance surveillance and monitoring in resource-limited settings. PloS one. 2011;6(11):e28184.

16. Murphy EL, Collier AC, Kalish LA, et al. Highly active antiretroviral therapy decreases mortality and morbidity in patients with advanced HIV disease. Ann Intern Med. 2001;135(1):17-26.

17. Montaner JS, Lima VD, Harrigan PR, et al. Expansion of HAART coverage is associated with sustained decreases in HIV/AIDS morbidity, mortality and HIV transmission: the "HIV Treatment as Prevention" experience in a Canadian setting. PloS one. 2014;9(2):e87872.

18. Gulick RM. Causes of HIV treatment failure. Combination Therapy of AIDS. USA: Springer; 2004. p. 159-194.

19. Gholami M, Sadeghi L, Baesi K, et al. Survey of antiretroviral drug resistance pattern among HIV-infected patients with treatment failure in Iran. J Hum Virol Retrovir. 2015;3(1):1-6.

20. Baesi K, Moallemi S, Farrokhi M, et al. Subtype classification of Iranian HIV-1 sequences registered in the HIV databases, 2006-2013. PloS one. 2014;9(9):e105098. 